\title{
Dynamic Output Feedback Robust Model Predictive Control via Zonotopic Set-Membership Estimation for Constrained Quasi-LPV Systems
}

\author{
Xubin Ping ${ }^{1}$ and Ning Sun ${ }^{2}$ \\ ${ }^{1}$ School of Electro-Mechanical Engineering, Xidian University, Xian 710071, China \\ ${ }^{2}$ School of Electronic Engineering, Xidian University, Xian 710071, China \\ Correspondence should be addressed to Xubin Ping; xbping@xidian.edu.cn
}

Received 5 July 2014; Revised 11 September 2014; Accepted 7 October 2014

Academic Editor: Dewei Li

Copyright ( $\odot 2015$ X. Ping and N. Sun. This is an open access article distributed under the Creative Commons Attribution License, which permits unrestricted use, distribution, and reproduction in any medium, provided the original work is properly cited.

For the quasi-linear parameter varying (quasi-LPV) system with bounded disturbance, a synthesis approach of dynamic output feedback robust model predictive control (OFRMPC) is investigated. The estimation error set is represented by a zonotope and refreshed by the zonotopic set-membership estimation method. By properly refreshing the estimation error set online, the bounds of true state at the next sampling time can be obtained. Furthermore, the feasibility of the main optimization problem at the next sampling time can be determined at the current time. A numerical example is given to illustrate the effectiveness of the approach.

\section{Introduction}

Model predictive control (MPC) or receding horizon control is a class of optimization based control methods, which explicitly utilizes process model parameters and measurements to optimize a performance function. At the current time, the optimization problem is carried out and then generates the optimal control input sequence. However, only the first element of control inputs is implemented. At the next sampling time, the output measurement is updated and the prediction horizon is shifted one step forward, and then the optimization problem is repeated. Due to its ability to handle the systems with hard constraints, it has attracted many researchers' attention, from both academic community and industrial society, for example, [1-4]. However, in real processes, the precise model parameters are seldomly available. Hence, robust MPC (RMPC) is more practical for real applications.

Linear parameter varying (LPV) systems are the systems whose parameters take their values in the prespecified sets and the dynamic characteristics depend on the time-varying parameters. When the time-varying parameters of LPV systems can be exactly known at the current time, the systems are called quasi-LPV (quasi-linear parameter varying) systems. It means that the model parameters are exactly known at the current time, but their future evolutions are uncertain and contained in the prescribed bounded sets. In RMPC, the model parametric uncertainty can be dealt with within the frame of LPV systems. For the online RMPC, at each time, a min-max optimization problem is often utilized to minimize the performance function of LPV systems, which considers all the possible realization of model parametric uncertainty [5]. Reference [6] extends the approaches in [5] to the quasiLPV system with a quasi-min-max optimization. In $[7,8]$, the controller designment considers the time-varying parameters of quasi-LPV systems having the bounds on their rate of variation. Reference [9] considers the quasi-LPV systems with a parameter-dependent control law.

However, in [5-9], the true states are assumed to be known and the bounded disturbance is not considered. In real processes, the true states are usually unmeasurable, and only the outputs with disturbance are available. In these situations, output feedback RMPC (OFRMPC) is more practical than the state feedback one for real applications. For OFRMPC with bounded disturbance and without model parametric uncertainty, see $[10,11]$. For OFRMPC with model parametric 
uncertainty and without bounded disturbance, see [12-14]. For OFRMPC with both polytopic uncertainty and bounded disturbance, see [15-20].

For OFRMPC, the bounds of estimation error set represent a kind of uncertainty, which has to be considered in the robust stability and physical constraints. When the model parametric uncertainty, bounds of estimation error set, and disturbance are known and contained in the bounded sets, the evolution of state estimation is a compact set which is consistent with the bounded uncertainties. The set-membership estimation considers nonstatistical description of model parameters, estimation error sets, and disturbance in the form of bounded uncertainties. When the set-membership estimation is utilized to state estimation, an appropriate representation of estimation error sets should consider the tradeoff between the precise representation of estimation error sets and the online computational burden. Polytopes can be used to represent exactly the estimation error set. However, if the vertices of polytopes increase dramatically, the computational burden becomes quickly prohibitive [21]. Ellipsoids can overcome the drawback by efficient computation. However, the Minkowski sum of ellipsoids is not necessary an ellipsoid, and an outer approximation ellipsoid is often used to estimate the Minkowski sum of ellipsoids, which may lead to the conservatism of state estimation. In recent years, zonotopes (a particular class of polytopes) have received more attention because of their advantages of having better precision in comparison with ellipsoidal sets and less complexity compared to polyhedral sets. Hence, several setmembership estimation methods based on zonotopes [2225] have been proposed.

The present paper considers a synthesis approach of dynamic OFRMPC for the quasi-LPV system with bounded disturbance. The main optimization problem is similar to that in $[16,19]$, while the estimation error sets are represented by zonotopes. The main contribution is a combination between the main optimization problem that calculates control parameters and the set-membership estimation based on zonotopic computation. By properly refreshing the estimation error sets, it can obtain the precise bounds of true states at the next sampling time. Furthermore, the feasibility of the main optimization problem at the next sampling time can be determined by checking a feasible problem at the current time.

\section{Notations, Basic Definitions, and Properties}

For any vector $x$ and positive-definite matrix $W,\|x\|_{W}^{2} \triangleq$ $x^{\mathrm{T}} W x . x(i \mid k)$ is the value of $x$ at time $k+i$, predicted at time $k . I$ is the identity matrix with appropriate dimension. $\varepsilon_{M} \triangleq\left\{\xi \mid \xi^{\mathrm{T}} M \xi \leq 1\right\}$ denotes the ellipsoid associated with the symmetric positive-definite matrix $M$. All vector inequalities are interpreted in an element-wise sense. An element belonging to $\operatorname{Co}\{\cdot\}$ means that it is a convex combination of the elements in $\{\cdot\}$, with the scalar combing coefficients nonnegative and their sum equal to 1 . The symbol " $\star$ " induces a symmetric structure in the matrix inequalities. A value with superscript “*” means that it is the optimal solution of the optimization problem. The time-dependence $(k)$ of the MPC decision variables is often omitted for brevity.

Let an interval $[a ; b]$ be defined as the set $\{x: a \leq$ $x \leq b\}$. The unitary interval is $\mathbf{B}=[-1 ; 1]$. A box $\left(\left[a_{1} ; b_{1}\right], \ldots,\left[a_{n} ; b_{n}\right]\right)^{\mathrm{T}}$ is an interval vector. A unitary box in $\mathfrak{R}^{m}$, denoted by $\mathbf{B}^{m}$, is a box composed by $m$ unitary intervals. The Minkowski sum of two sets $X$ and $Y$ is defined by $X \oplus Y=$ $\{x+y: x \in X, y \in Y\}$.

Definition 1. A $m$-zonotope in $\mathfrak{R}^{n}$ can be defined as the linear image of a $m$-dimensional hypercube in $\mathfrak{R}^{n}$, where $m$ is the order of the zonotope and satisfies $m \geq n$. Given a vector $p \epsilon$ $\mathfrak{R}^{n}$ and a matrix $H \in \mathfrak{R}^{n \times m}$, a $m$-zonotope is the following set:

$$
Z=p \oplus H \mathbf{B}^{m}=\left\{x \in \mathfrak{R}^{n} \mid x=p+H z, z \in \mathbf{B}^{m}\right\} .
$$

This is the Minkowski sum of the $m$-segments defined by $m$ columns of matrix $H$ in $\mathfrak{R}^{n}$. The center of zonotope $Z$ is vector $p ; H=\left[h_{1}, \ldots, h_{i}, \ldots, h_{m}\right]$, with $h_{i} \in \mathfrak{R}^{n}$, $i \in\{1, \ldots, m\}$, are the generators of the zonotope. Some properties for zonotopic computation are given as follows.

Property 1 (the Minkowski sum of two zonotopes [22]). Given two zonotopes $Z_{1}=p_{1} \oplus H_{1} \mathbf{B}_{1}^{m} \in \mathfrak{R}^{n}$ and $Z_{2}=$ $p_{2} \oplus H_{2} \mathbf{B}_{2}^{m} \in \mathfrak{R}^{n}$, the Minkowski sum of two zonotopes is also a zonotope defined by $Z=Z_{1} \oplus Z_{2}=\left(p_{1}+p_{2}\right) \oplus$ $\left[\begin{array}{ll}H_{1} & H_{2}\end{array}\right] \mathbf{B}^{m_{1}+m_{2}} \in \mathfrak{R}^{n}$.

Property 2 (the image of a zonotope [22]). The image of a zonotope $Z=p \oplus H \mathbf{B}^{m} \in \mathfrak{R}^{n}$ by a linear mapping $K$ can be computed by a standard matrix product $K Z=(K p) \oplus$ $(K H) \mathbf{B}^{m} \in \mathfrak{R}^{n}$.

Property 3 (zonotope reduction [22, 23]). Given a zonotope $Z=p \oplus H \mathbf{B}^{m} \in \mathfrak{R}^{n}$ and integer $f$ with $n<f<m$, denote by $\widehat{H}$ the matrix resulting from the reordering of the columns of matrix $H$ in decreasing order of Euclidean norm and by $O \epsilon$ $\mathfrak{R}^{n \times n}$ a diagonal matrix, satisfying the following conditions:

$$
\begin{gathered}
\widehat{H}=\left[\widehat{h}_{1}, \ldots, \widehat{h}_{i}, \ldots, \widehat{h}_{m}\right], \quad \text { with }\left\|\widehat{h}_{i}\right\|_{2} \geq\left\|\widehat{h}_{i+1}\right\|_{2}, \\
O_{i i}=\sum_{j=f-n+1}^{m}\left|\widehat{H}_{i j}\right|, \quad \text { with } i=\{1, \ldots, n\},
\end{gathered}
$$

and then $Z \subseteq \widehat{Z} \triangleq p \oplus\left[\widehat{H}_{T} \quad O\right] \mathbf{B}^{f} \in \mathfrak{R}^{n}$, where $\widehat{H}_{T}$ is obtained from the first $f-n$ columns of matrix $\widehat{H}$.

Remark 2. From Property 3 , it can obtain that $\widehat{Z}$ is an outer approximation of $Z$ with a lower order, which can be utilized to estimate a zonotope with high order. In Section 4.2, by applying Property 3, the order of the estimation error set can be reduced.

Zonotopes are the special case of polytopes and can be represented by half-space representation (H-representation) and vertex representation (V-representation). The definitions are as follows. 
Definition 3 (H-representation of a polytope). For $q$ halfspaces, a convex polytope $P$ is the set:

$$
P=\left\{x \in \mathfrak{R}^{n} \mid G x \leq g, G \in \mathfrak{R}^{q \times n}, g \in \mathfrak{R}^{q \times 1}\right\} .
$$

Definition 4 (V-representation of a polytope). A polytope $P$ is the set constructed by the convex combination of finite vertices $\chi_{i} \in \mathfrak{R}^{n}, i \in\left\{1, \ldots, V_{P}\right\}$; that is,

$$
\begin{aligned}
P & =\operatorname{Co}\left\{\chi_{1}, \ldots, \chi_{V_{P}}\right\} \\
& =\left\{\sum_{i=1}^{V_{P}} \alpha_{i} \chi_{i} \mid \chi_{i} \in \mathfrak{R}^{n}, \alpha_{i} \in \mathfrak{R}, \alpha_{i} \geq 0, \sum_{i=1}^{V_{P}} \alpha_{i}=1\right\},
\end{aligned}
$$

where $V_{P}$ is the total number of vertices related to the $\mathrm{V}$ representation of polytope $P$. From Definition 1, the unitary box $\mathbf{B}^{m}$ in $\Re^{m}$ has $2^{m}$ vertices, and each vertex of $\mathbf{B}^{m}$ is a $m$ dimensional vector with the element being 1 or -1 . Hence, according to (1), at most $2^{m}$ vertices of zonotope $Z$ can be calculated. We define the vertex which is the interior point of $Z$ as redundant vertex. To represent the zonotope $Z$ by $\mathrm{V}$ representation through $V_{Z}$ vertices, a Quickhull Algorithm in [26] can be employed to remove the redundant vertices. When the dimension $n$ of zonotope $Z$ is fixed, the relationship between $V_{Z}$ and $m$ can be obtained; for example, when $n=2$, $V_{Z}=2 m ; n=3, V_{Z}=m(m-1)+2$, respectively [27]. For high-dimensional systems, although the relationship between $V_{Z}$ and $m$ is complex, the upper bound of $V_{Z}$ can be calculated in polynomial time for the fixed dimension of zonotopes [28].

Figure 1 shows a zonotope $\bar{Z}=p \oplus H \mathbf{B}^{3}$, where $p=\left[\begin{array}{c}6 \\ 5\end{array}\right]$, $H=\left[\begin{array}{ccc}2.5 & -2 & 0.5 \\ 0 & -1 & -2\end{array}\right], m=3$; the three generators are $h_{1}=\left[\begin{array}{c}2.5 \\ 0\end{array}\right]$, $h_{2}=\left[\begin{array}{c}-2 \\ -1\end{array}\right], h_{3}=\left[\begin{array}{c}0.5 \\ -2\end{array}\right]$, which are the three columns of matrix $H$, respectively [27]. Based on Definition 1, the vertices of zonotope $\bar{Z}$ can be calculated for the different $2^{3}$ vertices of unitary box $\mathbf{B}^{3}$, where the different combinations of “+” and "-" denote the corresponding vertices of $\bar{Z}$. In order to describe the zonotope $\bar{Z}$ by V-representation through 6 $\left(V_{z}=2 \times 3\right)$ vertices, the redundant vertices denoted by " $(-,-,+)$ " and " $(+,+,-)$ " are removed.

\section{Problem Statement}

Consider the following discrete-time uncertain LPV system:

$$
\begin{gathered}
x(k+1)=A(k) x(k)+B(k) u(k)+D(k) w(k), \\
y(k)=C(k) x(k)+E(k) w(k),
\end{gathered}
$$

where $u \in \mathfrak{R}^{n_{u}}, x \in \mathfrak{R}^{n_{x}}, y \in \mathfrak{R}^{n_{y}}$, and $w \in \mathfrak{R}^{n_{w}}$ are input, state, output, and disturbance, respectively.

We make the following assumptions on system (6).

(A1) The time-varying system matrices $[A|B| C|D|$ $E](k)$ are known to vary within a polytope $\Omega$, that is, $[A|B| C|D| E](k) \in \Omega \triangleq \operatorname{Co}\left\{\left[A_{l}\left|B_{l}\right| C_{l} \mid\right.\right.$ $\left.\left.D_{l} \mid E_{l}\right], l=\{1, \ldots, L\}\right\}$, and there exist nonnegative coefficients $\lambda_{l}(k)$, where $\lambda_{l}(k)$ 's are exactly known at current time $k$, such that $\sum_{l=1}^{L} \lambda_{l}(k)=1$ and $[A|B|$ $C|D| E](k)=\sum_{l=1}^{L} \lambda_{l}(k)\left[A_{l}\left|B_{l}\right| C_{l}\left|D_{l}\right| E_{l}\right]$.

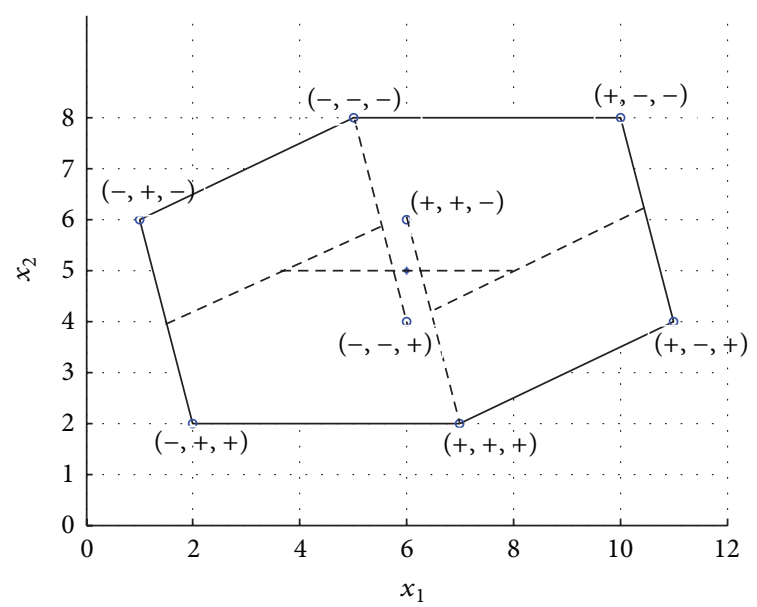

FIGURE 1: Representation of a zonotope $\bar{Z}$ by generators and vertices.

(A2) The bounded disturbance satisfies $w(k) \in \varepsilon_{P_{w}}$, and an outer approximation of the bounded ellipsoidal disturbance set can be represented by a zonotope $\mathbb{D}$; that is,

$\varepsilon_{P_{w}} \subseteq \mathbb{D} \triangleq 0 \oplus H_{w} \mathbf{B}^{d}, \quad H_{w} \in \mathfrak{R}^{n_{w} \times d}, d \geq n_{w}$,

where matrix $H_{w}$ is constructed by $d$ generators of zonotope $\mathbb{D}$.

(A3) The input and output constraints are required to satisfy

$$
-\bar{u} \leq u(k) \leq \bar{u}, \quad-\bar{\psi} \leq \Psi y(k+1) \leq \bar{\psi},
$$

where $\bar{u}=\left[\bar{u}_{1}, \bar{u}_{2}, \ldots, \bar{u}_{n_{u}}\right]^{\mathrm{T}} ; \bar{u}_{j}>0, j \in\left\{1, \ldots, n_{u}\right\}$; $\bar{\psi}=\left[\bar{\psi}_{1}, \bar{\psi}_{2}, \ldots, \bar{\psi}_{q}\right]^{\mathrm{T}} ; \bar{\psi}_{j}>0, j \in\{1, \ldots, q\} ; \Psi \in$ $\mathfrak{R}^{q \times n_{y}}$.

For the system (6), the dynamic output feedback controller is of the following form $[16,19]$ :

$$
\begin{aligned}
x_{c}(i+1 \mid k) & =A_{c}(i \mid k) x_{c}(i \mid k)+B_{c}(i \mid k) y(i \mid k), \\
u(i \mid k) & =C_{c}(i \mid k) x_{c}(i \mid k)+D_{c}(i \mid k) y(i \mid k),
\end{aligned}
$$

where $x_{c} \in \mathfrak{R}^{n_{x}},\left\{A_{c}(i \mid k), B_{c}(i \mid k)\right\}$ are the controller gain matrices, and $\left\{C_{c}(i \mid k), D_{c}(i \mid k)\right\}$ are the feedback gain matrices. The controller parameters $\left\{A_{c}(i \mid k), B_{c}(i \mid k), C_{c}(i \mid\right.$ $\left.k), D_{c}(i \mid k)\right\}$ take the parameter-dependent form as

$$
\begin{gathered}
A_{c}(i \mid k)=\sum_{l=1}^{L} \sum_{j=1}^{L} \lambda_{l}(k+i) \lambda_{j}(k+i) \bar{A}_{c}^{l j}, \\
D_{c}(i \mid k)=\bar{D}_{c}, \\
B_{c}(i \mid k)=\sum_{l=1}^{L} \lambda_{l}(k+i) \bar{B}_{c}^{l}, \\
C_{c}(i \mid k)=\sum_{j=1}^{L} \lambda_{j}(k+i) \bar{C}_{c}^{j} .
\end{gathered}
$$


Hence, the augmented closed-loop system, based on (9) and the predictions made by (6), is

$$
\begin{aligned}
\tilde{x}(i+1 \mid k)= & \Phi(i, k) \tilde{x}(i \mid k) \\
& +\Gamma(i, k) w(k+i), \quad \forall i \geq 0, \\
& \tilde{x}(0 \mid k)=\tilde{x}(k),
\end{aligned}
$$

where

$$
\begin{gathered}
\tilde{x}=\left[x^{\mathrm{T}}, x_{c}^{\mathrm{T}}\right]^{\mathrm{T}}, \\
\Phi_{l j}=\left[\begin{array}{cc}
A_{l}+B_{l} \bar{D}_{c} C_{j} & B_{l} \bar{C}_{c}^{j} \\
\bar{B}_{c}^{l} C_{j} & \bar{A}_{c}^{l j}
\end{array}\right], \\
\Gamma_{l j}=\left[\begin{array}{c}
B_{l} \bar{D}_{c} E_{j}+D_{l} \\
\bar{B}_{c}^{l} E_{j}
\end{array}\right], \\
{[\Phi(i, k) \mid \Gamma(i, k)]=\sum_{l=1}^{L} \sum_{j=1}^{L} \lambda_{l}(k+i) \lambda_{j}(k+i)\left[\Phi_{l j} \mid \Gamma_{l j}\right] .}
\end{gathered}
$$

At each time $k$, the true state $x(k)$ is unmeasurable. Hence it is necessary to use its bounds to represent the true state. Suppose, at time $k, x(k)$ is confined by a $m(k)$ order zonotope represented by

$$
\begin{array}{r}
x(k) \in X(k)=x_{c}(k) \oplus H(k) \mathbf{B}^{m(k)}, \\
H(k) \in \mathfrak{R}^{n_{x} \times m(k)}, \quad m(k) \geq n_{x},
\end{array}
$$

and then by applying Definition 1 and removing the redundant vertices, the true state $x(k)$ can be represented by the following convex set:

$$
x(k) \in \operatorname{Co}\left\{\chi_{1}(k), \chi_{2}(k), \ldots, \chi_{V_{X(k)}}(k)\right\},
$$

where $\chi_{r}(k), r \in\left\{1,2, \ldots, V_{X(k)}\right\}$, are the vertices of $X(k)$ and $V_{X(k)}$ is the total number of vertices related to the $\mathrm{V}$ representation of zonotope $X(k)$ at time $k$.

\section{Synthesis Approach of Dynamic OFRMPC}

4.1. The Main Optimization Problem. In the dynamic OFRMPC approach, at each time $k$, based on the dynamic OFRMPC approaches in $[16,19]$, the following main optimization problem is considered:

$$
\begin{aligned}
& \min _{\gamma, \mathrm{Q}, \vec{A}_{c}^{l j}, \vec{B}_{c}, \bar{C}_{c}^{j}, \bar{D}_{c}[A|B| C|D| E](k+i) \in \Omega, w(k+i) \in \varepsilon_{P_{w}}} \max _{\text {s.t. } \quad} \quad \tilde{x}(k) \in \varepsilon_{Q^{-1}}, \\
& \quad\|\tilde{x}(i \mid k)\|_{Q^{-1}}^{2} \\
& \geq 1 \Longrightarrow\|\tilde{x}(i \mid k)\|_{Q^{-1}}^{2}-\|\tilde{x}(i+1 \mid k)\|_{Q^{-1}}^{2} \\
& \quad \geq \frac{1}{\gamma}\left[\|y(i \mid k)\|_{\mathscr{Q}}^{2}+\|u(i \mid k)\|_{\mathscr{R}}^{2}\right],
\end{aligned}
$$

$$
\forall i \geq 0
$$

$$
\begin{aligned}
& -\bar{u} \leq u(i \mid k) \leq \bar{u}, \quad \forall i \geq 0, \\
& -\bar{\psi} \leq \Psi y(i+1 \mid k) \leq \bar{\psi}, \quad \forall i \geq 0,
\end{aligned}
$$

where $\gamma$ is the performance cost, (16) is the augmented state constraint, (17) is the stability/optimality condition, (18) is the input constraint, (19) is the output constraint, the matrices $Q$, $\mathscr{R}$ are the positive-definite weighting matrices, and $Q$ satisfies the following conditions:

$$
\begin{gathered}
Q^{-1}=M=\left[\begin{array}{ll}
M_{1} & M_{2}^{\mathrm{T}} \\
M_{2} & M_{3}
\end{array}\right], \\
Q=\left[\begin{array}{ll}
Q_{1} & Q_{2}^{\mathrm{T}} \\
Q_{2} & Q_{3}
\end{array}\right] .
\end{gathered}
$$

Choosing $M_{2}=-M_{1}$, then $M_{3}=M_{1}\left(M_{1}-Q_{1}^{-1}\right) M_{1}, Q_{2}=$ $Q_{3}=Q_{1}-M_{1}^{-1}$.

In the following, some previous results mainly in $[16$, 19] are reviewed to deal with constraint conditions (16)(19). By applying the definition of augmented state and (14), constraint (16) holds if there exist positive-definite matrices $\left\{M_{1}, Q_{1}, \bar{M}_{3}, M_{4}\right\}$ such that

$$
\begin{gathered}
{\left[\begin{array}{l}
\chi_{r}(k) \\
x_{c}(k)
\end{array}\right]^{\mathrm{T}}\left[\begin{array}{cc}
M_{1} & -M_{1} \\
-M_{1} & \bar{M}_{3}
\end{array}\right]\left[\begin{array}{l}
\chi_{r}(k) \\
x_{c}(k)
\end{array}\right] \leq 1,} \\
r \in\left\{1,2, \ldots, V_{X(k)}\right\}, \\
{\left[\begin{array}{cc}
\bar{M}_{3} & -M_{1} \\
-M_{1} & M_{4}
\end{array}\right] \geq 0} \\
{\left[\begin{array}{cc}
M_{1}-M_{4} & I \\
I & Q_{1}
\end{array}\right] \geq 0 .}
\end{gathered}
$$

For all $\tilde{x}(i \mid k)$ and all admissible $w(k+i)$, condition (17) holds if there exist a scalar $\alpha$ and matrices $\left\{\widehat{A}_{c}^{l j}, \widehat{B}_{c}^{l}, \widehat{C}_{c}^{j}, \widehat{D}_{c}\right\}$ such that

$$
\Upsilon^{\mathrm{QB}}(i, k)=\sum_{l=1}^{L} \sum_{j=1}^{L} \lambda_{l}(k+i) \lambda_{j}(k+i) \Upsilon_{l j}^{\mathrm{QB}} \geq 0, \quad \forall i \geq 0
$$

$$
\Upsilon_{l j}^{\mathrm{QB}}
$$$$
=\left[\begin{array}{ccccccc}
(1-\alpha) M_{1} & \star & \star & \star & \star & \star & \star \\
(1-\alpha) I & (1-\alpha) Q_{1} & \star & \star & \star & \star & \star \\
0 & 0 & \alpha P_{w} & \star & \star & \star & \star \\
A_{l}+B_{l} \widehat{D}_{c} C_{j} & A_{l} Q_{1}+B_{l} \widehat{C}_{c}^{j} & B_{l} \widehat{D}_{c} E_{j}+D_{l} & Q_{1} & \star & \star & \star \\
M_{1} A_{l}+\widehat{B}_{c}^{l} C_{j} & \widehat{A}_{c}^{j} & \widehat{B}_{c}^{l} E_{j}+M_{1} D_{l} & I & M_{1} & \star & \star \\
Q^{1 / 2} C_{j} & Q^{1 / 2} C_{j} Q_{1} & \widehat{Q}^{1 / 2} E_{j} & 0 & 0 & \gamma I & \star \\
\mathscr{R}^{1 / 2} \widehat{D}_{c} C_{j} & \mathscr{R}^{1 / 2} \widehat{C}_{c}^{j} & \mathscr{R}^{1 / 2} \widehat{D}_{c} E_{j} & 0 & 0 & 0 & \gamma I
\end{array}\right],
$$ 
with (10) being parameterized as

$$
\begin{gathered}
\bar{D}_{c}=\widehat{D}_{c}, \\
\bar{C}_{c}^{j}=\left(\bar{D}_{c} C_{j} Q_{1}-\widehat{C}_{c}^{j}\right)\left(M_{1}^{-1}-Q_{1}\right)^{-1}, \\
\bar{B}_{c}^{l}=M_{1}^{-1}\left(M_{1} B_{l} \bar{D}_{c}-\widehat{B}_{c}^{l}\right),
\end{gathered}
$$

$$
\begin{aligned}
\bar{A}_{c}^{l j}= & \left(M_{1}^{-1} \widehat{A}_{c}^{l j}-A_{l} Q_{1}-B_{l} \bar{D}_{c} C_{j} Q_{1}+\bar{B}_{c}^{l} C_{j} Q_{1}\right) \\
& \times\left(M_{1}^{-1}-Q_{1}\right)^{-1}+B_{l} \bar{C}_{c}^{j} .
\end{aligned}
$$

Suppose, at time $k$, constrains (21), (22), and (23) are satisfied; there exist positive-definite matrices $\{U, \widetilde{\Xi}\}$ such that

$$
\begin{aligned}
& \Upsilon_{j}^{u}=\left[\begin{array}{cccc}
M_{1} & \star & \star & \star \\
I & Q_{1} & \star & \star \\
0 & 0 & P_{w} & \star \\
\sqrt{2} \widehat{D}_{c} C_{j} & \sqrt{2} \widehat{C}_{c}^{j} & \sqrt{2} \widehat{D}_{c} E_{j} & U
\end{array}\right] \geq 0 \\
& U_{s s} \leq \bar{u}_{s}^{2}, \quad j=1, \ldots, L, \quad s=1, \ldots, n_{u} \text {, } \\
& \Upsilon^{h}(i, k)=\sum_{l=1}^{L} \sum_{j=1}^{L} \lambda_{l}(k+i) \lambda_{j}(k+i) \Upsilon_{l j}^{h} \geq 0, \\
& h=1, \ldots, L, \quad \forall i \geq 0, \\
& Y_{l j}^{h}=\left[\begin{array}{cccc}
M_{1} & \star & \star & \star \\
I & Q_{1} & \star & \star \\
0 & 0 & P_{w} & \star \\
\Psi C_{h}\left(A_{l}+B_{l} \widehat{D}_{c} C_{j}\right) & \Psi C_{h}\left(A_{l} Q_{1}+B_{l} \widehat{C}_{c}^{j}\right) & \Psi C_{h}\left(B_{l} \widehat{D}_{c} E_{j}+D_{l}\right) & \widetilde{\Xi}
\end{array}\right] \text {, } \\
& \widetilde{\Xi}_{s s} \leq \widetilde{\psi}_{s}^{2}, \quad s=1, \ldots, q, \\
& \widetilde{\psi}_{s}=\frac{1}{\sqrt{2}}\left(\bar{\psi}_{s}-\sqrt{\zeta_{s}}\right), \\
& \zeta_{s}=\max _{h}\left(\Psi E_{h} P_{w}^{-1} E_{h}^{\mathrm{T}} \Psi^{\mathrm{T}}\right)_{s s},
\end{aligned}
$$

where $U_{s s}\left(\widetilde{\Xi}_{s s}\right)$ is the $s$ th diagonal element of $U(\widetilde{\Xi})$, and then by applying (10) and (24), the constraints (18) and (19) are satisfied.

From the above conditions, it is shown that the main optimization problem (15)-(19) can be approximated by

$$
\min _{\gamma, \alpha, Q_{1}, M_{1}, \bar{M}_{3}, M_{4}, \widehat{A}_{c}^{l j}, \widehat{B}_{c}^{l}, \widehat{C}_{c}^{j}, \widehat{D}_{c}, U, \widetilde{\Xi}} \gamma,
$$

s.t. $(21),(22),(23),(25)$.

At each time $k$, if the main optimization problem (26) is solved, then (11) is quadratically bounded $[29,30]$ within a common Lyapunov matrix $Q^{-1}$, and the controller parameters are calculated by (10) and (24). The main optimization problem (26) can be solved by an LMI (linear matrix inequality) tool, which is the same as in $[16,19]$.

Remark 5. In [16], the true state constraint in the main optimization problem is represented by the V-representation of general polyhedral sets. In [19], to guarantee the recursive feasibility of the optimization problem based on polyhedral bounds, the true state constraint in the main optimization problem utilizes either the ellipsoidal set or the polyhedral set, with the latter being used if and only if it is contained in the former. Compared with $[16,19]$, the present paper utilizes the V-representation of zonotopes to represent the bounds of true state.

4.2. The Refreshment of Estimation Error Sets. Define the estimation error $e(k) \triangleq x(k)-x_{c}(k)$. At each time $k$, for the quasi-LPV system (6), the model parameters $[A|B|$ $C \quad|\quad D| E](k)$ are exactly known at the current time $k$. If the controller parameters $\left\{A_{c}(k), B_{c}(k), C_{c}(k), D_{c}(k)\right\}$ are obtained, then according to (11) and the definition of estimation error, it is shown that

$$
\begin{aligned}
e(k+1)= & \sum_{l=1}^{L} \sum_{j=1}^{L} \lambda_{l}(k) \lambda_{j}(k) \\
& \times\left\{\left(A_{l}+B_{l} \bar{D}_{c} C_{j}-\bar{B}_{c}^{l} C_{j}\right) x(k)\right. \\
& +\left(B_{l} \bar{C}_{c}^{j}-\bar{A}_{c}^{l j}\right) x_{c}(k) \\
& \left.+\left(B_{l} \bar{D}_{c} E_{j}+D_{l}-\bar{B}_{c}^{l} E_{j}\right) w(k)\right\}
\end{aligned}
$$




$$
\begin{aligned}
= & {\left[A(k)+B(k) D_{c}(k) C(k)-B_{c}(k) C(k)\right] x(k) } \\
& +\left[B(k) C_{c}(k)-A_{c}(k)\right] x_{c}(k) \\
& +\left[B(k) D_{c}(k) E(k)+D(k)-B_{c}(k) E(k)\right] w(k) .
\end{aligned}
$$

By applying (7) and (13) and Properties 1 and 2, it can be obtained that

$$
\begin{aligned}
e(k+1) \in \mathscr{E}(k+1) \\
\triangleq\left[A(k)+B(k) D_{c}(k) C(k)\right. \\
\left.\quad-B_{c}(k) C(k)+B(k) C_{c}(k)-A_{c}(k)\right] x_{c}(k) \\
\oplus\left\{\left[A(k)+B(k) D_{c}(k) C(k)-B_{c}(k) C(k)\right] H(k),\right. \\
\left.\quad\left[B(k) D_{c}(k) E(k)+D(k)-B_{c}(k) E(k)\right] H_{w}\right\} \\
\times \mathbf{B}^{m(k)+d} .
\end{aligned}
$$

From (28), the estimation error set at time $k+1$ can be represented by a $[m(k)+d]$-zonotope $\mathscr{E}(k+1)=p(k+1) \oplus$ $\bar{H}(k+1) \mathbf{B}^{m(k)+d}$, where

$$
\begin{aligned}
& p(k+1) \\
& =\left[A(k)+B(k) D_{c}(k) C(k)\right. \\
& \left.\quad-B_{c}(k) C(k)+B(k) C_{c}(k)-A_{c}(k)\right] x_{c}(k), \\
& \bar{H}(k+1) \\
& =\left[\left[A(k)+B(k) D_{c}(k) C(k)-B_{c}(k) C(k)\right] H(k),\right. \\
& \left.\quad\left[B(k) D_{c}(k) E(k)+D(k)-B_{c}(k) E(k)\right] H_{w}\right] .
\end{aligned}
$$

It can easily be obtained from (28) that, with the evolution of time, the order of zonotope $\mathscr{E}(k+1)$ will increase. Accordingly, the generators and the vertices of zonotope $\mathscr{E}(k+1)$ will increase, which may lead to more LMIs in the main optimization problem (26) to enforce the augmented state constraints and then increase the online computational burden of the main optimization problem (26). To decrease the online computational burden of the main optimization problem (26), we impose the restriction on the order of $\mathscr{E}(k+$ $1)$ by $m(k)+d \leq N_{z}$. When $m(k)+d>N_{z}$, Property 3 is applied to construct a low order $N_{z}$-zonotope $\widehat{\mathscr{E}}(k+1)=$ $p(k+1) \oplus \widehat{H}(k+1) \mathbf{B}^{N_{z}}$ for outer approximation of the high order zonotope $\mathscr{E}(k+1)$; that is, $\mathscr{E}(k+1) \subseteq \widehat{\mathscr{E}}(k+1)$, where

$$
\begin{array}{r}
\widehat{H}(k+1)=\left[\widehat{H}_{T}(k+1)\right. \\
\widehat{H}(k+1) \in \Re^{n_{x} \times N_{z}},
\end{array}
$$

and $\widehat{H}_{T}(k+1) \in \mathfrak{R}^{n_{x} \times\left[N_{z}-n_{x}\right]}$ and $O(k+1) \in \mathfrak{R}^{n_{x} \times n_{x}}$ are calculated according to (2) and (3), respectively. From the above derivations, it can be obtained that the estimation error set at time $k+1$ is one of the following forms:

$$
\begin{gathered}
P(k+1)=\mathscr{E}(k+1), \\
H(k+1)=\bar{H}(k+1), \\
m(k+1)=m(k)+d, \quad \text { when } m(k)+d \leq N_{z} ; \\
P(k+1)=\widehat{\mathscr{E}}(k+1), \\
H(k+1)=\widehat{H}(k+1), \\
m(k+1)=N_{z}, \quad \text { when } m(k)+d>N_{z} .
\end{gathered}
$$

When the estimation error set $P(k+1)$ is obtained, according to Definition 1 , at most $2^{m(k+1)}$ vertices can be calculated. By removing the redundant vertices, the set $P(k+$ 1) can be described by $\mathrm{V}$-representation through $V_{P(k+1)}$ vertices. The vertices of the true state set $X(k+1)$ are the same as $P(k+1)$; that is, $V_{X(k+1)}=V_{P(k+1)}$. Hence the $\mathrm{V}$ representation of the true state at time $k+1$ is

$$
\begin{gathered}
x(k+1) \in \operatorname{Co}\left\{\chi_{1}(k+1), \chi_{2}(k+1), \ldots, \chi_{V_{X(k+1)}}(k+1)\right\}, \\
\chi_{r}(k+1)=x_{c}(k+1)+v_{r}(k+1), \\
r \in\left\{1,2, \ldots, V_{X(k+1)}\right\} .
\end{gathered}
$$

Remark 6. In Section 2, we have mentioned the relationship between $V_{z}$ (the number of vertices related to zonotopes), $m$ (the order of zonotopes), and the dimension of zonotopes. For the refreshment of estimation error sets in Section 4.2, when the dimension of true state is $n_{x}=2, V_{X(k+1)}=V_{P(k+1)}=$ $2 m(k+1)$; when $n_{x}=3, V_{X(k+1)}=V_{P(k+1)}=m(k+1)[m(k+1)-$ $1]+2$, respectively [27]. For high-dimensional systems, when $n_{x}$ is fixed, the upper bound of $V_{X(k+1)}\left(\right.$ or $\left.V_{P(k+1)}\right)$ is related to $m(k+1)$ and can be calculated in polynomial time [28]. In [16], $V_{X(k+1)}$ (or $V_{P(k+1)}$ ) only depends on the dimension of true state; that is, $V_{X(k+1)}=V_{P(k+1)}=2^{n_{x}}$. In [19], the upper bound of $V_{X(k+1)}\left(\right.$ or $\left.V_{P(k+1)}\right)$ is bounded by properly selecting an integer $\widehat{p}$.

In $[16,19]$, the outer approximation of ellipsoidal bounded disturbance is represented by the $\mathrm{V}$-representation of the polytope. The present paper utilizes zonotopes to outer approximation of $\varepsilon_{P_{w}}$. The outer approximation of ellipsoids introduces conservatism. However, by properly increasing the vertices of outer approximation polyhedral sets in [16, 19] and choosing matrix $H_{w}$ with proper generators, the conservatism can be reduced.

4.3. The Feasibility of the Optimization Problem at the Next Sampling Time. The original optimization problem (15)-(19) is recursive feasible; that is, it is feasible for all $k>0$ if and only if it is feasible at time $k=0$. However, by using the bounds of true state to handle the uncertainty of true state, the recursive feasibility may be lost for the main optimization problem (26). If the main optimization 
problem (26) is solved at time $k$, then the optimal solutions $\left\{\alpha, \gamma, \widehat{A}_{c}^{l j}, \widehat{B}_{c}^{l}, \widehat{C}_{c}^{j}, \widehat{D}_{c}, Q_{1}, M_{1}, U, \widetilde{\Xi}\right\}^{*}(k)$ are obtained, and $\widetilde{x}(k) \in \varepsilon_{\left[Q^{*}(k)\right]^{-1}}$. At time $k+1$, by choosing

$$
\begin{gathered}
M_{4}(k+1)=M_{1}^{*}(k)-Q_{1}^{*}(k)^{-1}, \\
M_{1}(k+1)=M_{1}^{*}(k), \\
Q_{1}(k+1)=Q_{1}^{*}(k), \\
\bar{M}_{3}(k+1)=M_{3}(k+1) \\
=M_{1}^{*}(k)\left[M_{1}^{*}(k)-Q_{1}^{*}(k)^{-1}\right]^{-1} M_{1}^{*}(k)^{\mathrm{T}},
\end{gathered}
$$

then (22) is satisfied as follows:

$$
\begin{gathered}
{\left[\begin{array}{cc}
\bar{M}_{3}(k+1) & -M_{1}(k+1) \\
-M_{1}(k+1) & M_{4}(k+1)
\end{array}\right] \geq 0,} \\
{\left[\begin{array}{cc}
M_{1}(k+1)-M_{4}(k+1) & I \\
I & Q_{1}(k+1)
\end{array}\right] \geq 0 .}
\end{gathered}
$$

Based on (33) and (34) and the definitions of $Q$ and $M$, then, by applying the Schur complement, it can be obtained that if the problem (35) is feasible:

$$
\begin{aligned}
& \text { find } \begin{array}{l}
\eta, \\
\text { s.t. }\left[\begin{array}{ccc}
\eta & \star & \star \\
\chi_{r}(k+1) & Q_{1}^{*}(k) & \star \\
x_{c}(k+1) & Q_{2}^{*}(k) & Q_{3}^{*}(k)
\end{array}\right] \geq 0, \\
0<\eta \leq 1, \quad r \in\left\{1,2 \ldots, V_{X(k+1)}\right\},
\end{array}
\end{aligned}
$$

then (21) is satisfied and $\widetilde{x}(k+1) \in \varepsilon_{\left[Q^{*}(k)\right]^{-1}}$. In the main optimization problem (26), only (21) and (22) are related to the process state information. By further choosing $\left\{\alpha, \gamma, \widehat{A}_{c}^{l j}, \widehat{B}_{c}^{l}, \widehat{C}_{c}^{j}, \widehat{D}_{c}, U, \widetilde{\Xi}\right\}(k+1)=$ $\left\{\alpha, \gamma, \widehat{A}_{c}^{l j}, \widehat{B}_{c}^{l}, \widehat{C}_{c}^{j}, \widehat{D}_{c}, U, \widetilde{\Xi}\right\}^{*}(k)$, the feasible solutions for the main optimization problem (26) at time $k+1$ can be constructed. The feasible solutions for (21) at time $k+1$ can be directly constructed through (33). Hence, the feasibility of (35) determines not only whether $\widetilde{x}(k+1)$ is contained in the ellipsoidal set $\varepsilon_{\left[Q^{*}(k)\right]^{-1}}$ or not, but also the feasibility of the main optimization problem (26) at time $k+1$.

Remark 7. In [16], the refreshment of estimation error sets and the feasibility of the main optimization problem at the next sampling time are performed simultaneously by an iterative auxiliary optimization problem. Furthermore, it involves the $\mathrm{H}$-representation of estimation error set in the auxiliary optimization problem. In [19], to guarantee the recursive feasibility of the main optimization problem based on polyhedral sets, the recursions of both polyhedral sets and ellipsoidal sets are obtained. At the next sampling time, the polyhedral set is used in the main optimization problem if and only if it is contained in the ellipsoidal set. The present paper refreshes the estimation error set via zonotopic computations and is represented by V-representation. Then problem (35) is applied to determine whether the main optimization problem (26) is solved or not at the next sampling time.

\subsection{Overall Solutions}

Algorithm 8. Choose a feasible $x_{c}(0)$ and the initial constrain of $x(0)$ represented by a zonotope $x_{c}(0) \oplus H(0) \mathbf{B}^{m(0)}$, where $H(0) \in \mathfrak{R}^{n_{x} \times m(0)}$. Let $p_{\text {ro }}=1$. Select the maximal order of zonotope related to the estimation error set as $N_{z}$. At each time $k \geq 0$, perform the following steps.

(1) If $p_{\mathrm{ro}}=1$, then solve the main optimization problem (26) and obtain the controller parameters by (10) and (24).

(2) If $p_{\mathrm{ro}}=0$, then inherit the previous controller parameters.

(3) Implement $u(k)=C_{c}(k) x_{c}(k)+D_{c}(k) y(k)$, and calculate $x_{c}(k+1)=A_{c}(k) x_{c}(k)+B_{c}(k) y(k)$.

(4) Calculate $\mathscr{E}(k+1)$ by (28). If $m(k)+d \leq N_{z}$, then let $P(k+1)=\mathscr{E}(k+1)$; else then calculate $\widehat{\mathscr{E}}(k+1)$ by (30) and let $P(k+1)=\widehat{\mathscr{E}}(k+1)$.

(5) Check the feasibility of problem (35). If problem (35) is feasible, then let $p_{\text {ro }}=1$; else then let $p_{\text {ro }}=0$.

The complexity analysis of Algorithm 8 and the related algorithms for worst cases are listed in Table 1. For the main optimization problem in Table 1, an LMI tool is utilized. The complexity for solving the main optimization problem is polynomial time, which (regarding the fastest interior-point algorithms) is proportional to $\mathfrak{\Re}^{3} \mathfrak{Q}$, where $\mathbb{\mathfrak { R }}$ is the number of scalar LMI variables and $\mathbb{Q}$ is the number of scalar LMI rows (see [31]). The complexities of Algorithm 1 in [16], Algorithm 1 in [19], and Algorithm 8 are summarized in Table 1. From Table 1, it can be obtained that the computational burden of the main optimization problem is different mainly in how many of scalar LMI rows are utilized to describe the bounds of estimation error (or true state). In the main optimization problem of [16], $\mathfrak{Q}$ depends on the dimension of true state $n_{x}$; $\mathcal{Q}$ in the main optimization problem of [19] can be confined by properly choosing $\widehat{p}$. In Algorithm 8 , the restriction on the order of zonotope by $N_{z}$ can limit $V_{X(k)}$.

For the auxiliary optimization problem in [16], the computational burden mainly comes from an iterative method. Algorithm 1 in [19] and Algorithm 8 take the advantage of Quickhull Algorithm in [26] to remove the redundant vertices and obtain the estimation error set by $\mathrm{V}$-presentation. The worst-case complexity of Quickhull in [26] is $O\left(V \log r_{p}\right)$ for $n_{x} \leq 3$ and $O\left(V f_{r} / r_{p}\right)$ for $n_{x} \geq 4$, where $V$ is the number of input points in $\mathfrak{R}^{n_{x}}, r_{p}$ is the number of processed points, and $f_{r}$ is the maximum number of facets for $r_{p}$ processed vertices, respectively. Furthermore, the outer approximation of ellipsoidal sets in [19] and problem (35) are solved by an LMI tool, and the complexity analysis of the related parameters $\mathfrak{K}$ and $\mathfrak{Q}$ is listed in Table 1 . 
TABLE 1: The complexity analysis of the compared algorithms.

\begin{tabular}{|c|c|c|}
\hline Different algorithms & Main optimization problem & $\begin{array}{l}\text { Refreshment of estimation error set and/or determination of } \\
\text { feasibility problem }\end{array}$ \\
\hline Algorithm 1 in [16] & $\mathfrak{K}=\mathfrak{\mathfrak { K }}_{M}, \mathfrak{Q}=\mathfrak{Q}_{M}+2^{n_{x}}$ & $\begin{array}{l}\text { Each iteration of }(35) \text { in }[16]: \mathfrak{K}=n_{x}\left(n_{x}+1\right)+1, \\
\mathfrak{Q}=n_{x}^{2}+2 n_{x} n_{p}^{w} 2^{n_{x}}+2^{n_{x}}\left(1+n_{x}\right)+3 n_{x}+1 ;(35) \text { in [16] is infeasible: } \\
\mathfrak{N}=1, \mathfrak{Q}=2 n_{x} n_{p}^{w} 2^{n_{x}}\end{array}$ \\
\hline Algorithm 1 in [19] & $\begin{array}{l}\text { For polyhedral set: } \mathfrak{K}=\mathfrak{\Re}_{M}, \mathfrak{Q}=\mathfrak{Q}_{M}+\widehat{p} \\
\text { for ellipsoidal set: } \mathfrak{\Re}=\mathfrak{\Re}_{M}+1 \\
\mathfrak{Q}=\mathfrak{Q}_{M}+3\end{array}$ & $\begin{array}{l}\text { Quickhull Algorithm: } O\left(\widehat{p} n_{p}^{w} \log r_{p}\right), n_{x} \leq 3 ; O\left(\widehat{p} n_{p}^{w} f_{r} / r_{p}\right), n_{x} \geq 4 \\
\text { outer approximation of ellipsoidal sets: } \mathbb{\Re}=\widehat{p}, \mathfrak{L}=\widehat{p}^{2} n_{p}^{w}\end{array}$ \\
\hline Algorithm 8 & $\mathfrak{K}=\mathfrak{\Re}_{M}, \mathfrak{Q}=\mathfrak{Q}_{M}+V_{X(k)}$ & $\begin{array}{l}\text { Quickhull Algorithm: } O\left(2^{N_{z}} \log r_{p}\right), n_{x} \leq 3 ; O\left(2^{N_{z}} f_{r} / r_{p}\right), n_{x} \geq 4 \text {; } \\
\text { problem (35): } \mathbb{K}=1, \mathfrak{Q}=2 n_{x}+1\end{array}$ \\
\hline
\end{tabular}

Theorem 9. For system (6), Algorithm 8 is applied. Suppose $x(0) \in X(0)=x_{c}(0) \oplus H(0) \mathbf{B}^{m(0)}$, where $H(0) \in \mathfrak{R}^{n_{x} \times m(0)}$. If the main optimization problem (26) is feasible at time $k=0$, then $\tilde{x}(k)$ will converge to a neighborhood of $\tilde{x}=0$, and the input and output constraints (8) are satisfied for all time $k \geq 0$.

Proof. The proof is similar to the "proof of Theorem 1" in [32]. Suppose, at time $k$, Algorithm 8 is performed with a refreshed control law; at time $k+1, k+2, \ldots, k+l-1, l>0$, it obtains $p_{\text {ro }}=0$, and at time $k+l$ it obtains $p_{\text {ro }}=1$. According to Algorithm 8, the optimal solutions to (26) at time $k$ will be feasible to $(26)$ at time $k+l$. Hence, $\gamma(k+l)=$ $\gamma^{*}(k)$ is a feasible bound of performance cost at time $k+l$. At time $k+l$, solve the main optimization problem (26), $\gamma^{*}(k+l) \leq \gamma(k+l)$ will be obtained, and then it will satisfy $\gamma^{*}(k+l) \leq \gamma^{*}(k)$. By induction, $\gamma^{*}\left(k^{o}\right)$ will not increase with time $k^{\mathrm{o}}$, where $k^{\mathrm{o}}$ is the time when the main optimization problem (26) is solved. With the evolution of time, $\gamma^{*}\left(k^{0}\right)$ will tend to a constant value denoted by $\gamma_{c}$, and the corresponding positively invariant ellipsoidal set related to $\gamma_{c}$ for system (11) is represented by ellipsoidal set $\varepsilon_{\mathrm{Q}_{c}^{-1}}$.

Consider the following uncorrupt system (i.e., the system is not corrupted by disturbance):

$$
\begin{gathered}
\tilde{x}_{\mathrm{u}}\left(i+1 \mid k^{\mathrm{o}}\right)=\Phi\left(i, k^{\mathrm{o}}\right) \tilde{x}_{\mathrm{u}}\left(i \mid k^{\mathrm{o}}\right), \quad \forall i \geq 0, \\
\tilde{x}_{\mathrm{u}}\left(0 \mid k^{\mathrm{o}}\right)=\tilde{x}\left(k^{\mathrm{o}}\right), \\
u_{\mathrm{u}}\left(i \mid k^{\mathrm{o}}\right)=C_{c}\left(i \mid k^{\mathrm{o}}\right) x_{c, \mathrm{u}}\left(i \mid k^{\mathrm{o}}\right)+D_{c}\left(i \mid k^{\mathrm{o}}\right) y_{\mathrm{u}}\left(i \mid k^{\mathrm{o}}\right), \\
y_{\mathrm{u}}\left(i \mid k^{\mathrm{o}}\right)=C\left(k^{\mathrm{o}}+i\right) x_{\mathrm{u}}\left(i \mid k^{\mathrm{o}}\right) .
\end{gathered}
$$

The quadratic boundedness condition (23) guarantees the asymptotic stability of the uncorrupt system (36). Hence,

$$
\begin{aligned}
& \left\|\tilde{x}_{\mathrm{u}}\left(i \mid k^{\mathrm{o}}\right)\right\|_{\mathrm{Q}^{-1}}^{2}-\left\|\tilde{x}_{\mathrm{u}}\left(i+1 \mid k^{\mathrm{o}}\right)\right\|_{\mathrm{Q}^{-1}}^{2} \\
& \quad \geq \frac{1}{\gamma\left(k^{\mathrm{o}}\right)}\left[\left\|y_{\mathrm{u}}\left(i \mid k^{\mathrm{o}}\right)\right\|_{\mathscr{Q}}^{2}+\left\|u_{\mathrm{u}}\left(i \mid k^{\mathrm{o}}\right)\right\|_{\mathscr{R}}^{2}\right],
\end{aligned}
$$

$\forall i \geq 0$
By summing (37) from $i=0$ to $i=\infty$, it achieves

$$
\begin{aligned}
J_{\infty}\left(k^{\mathrm{o}}\right) & =\sum_{i=0}^{\infty}\left[\left\|y_{\mathrm{u}}\left(i \mid k^{\mathrm{o}}\right)\right\|_{\mathscr{Q}}^{2}+\left\|u_{\mathrm{u}}\left(i \mid k^{\mathrm{o}}\right)\right\|_{\mathscr{R}}^{2}\right] \\
& \leq \gamma\left(k^{\mathrm{o}}\right)\left\|\tilde{x}\left(k^{\mathrm{o}}\right)\right\|_{Q^{-1}} \leq \gamma\left(k^{\mathrm{o}}\right) .
\end{aligned}
$$

Hence, $\gamma\left(k^{\circ}\right)$ is also an upper bound of performance cost for the uncorrupt system (36). The condition $J_{\infty}\left(k^{\circ}\right) \leq \gamma^{*}\left(k^{\circ}\right)$ results in $\lim _{i \rightarrow \infty} y_{\mathrm{u}}\left(i \mid k^{\mathrm{o}}\right)=0$ and $\lim _{i \rightarrow \infty} u_{\mathrm{u}}\left(i \mid k^{\mathrm{o}}\right)=$ 0 . When $\gamma^{*}\left(k^{\mathrm{o}}\right)$ tends to a constant value, $\gamma_{c}$ means that the augmented state will converge to a neighborhood of $\tilde{x}=0$ represented by $\varepsilon_{Q_{c}^{-1}}$ and stay in this neighborhood thereafter. Satisfaction of the input and output constraints (8) is due to (25).

\section{Numerical Example}

Consider the nonlinear continuous stirred tank reactor (CSTR) model, which has been studied in [12, 16, 19]. Similar to $[16,19]$, the nonlinear CSTR model is represented as the quasi-LPV system around the operation point, which considers the physical constraints and bounded disturbance. The details corresponding to system (6) are $L=4$,

$$
\begin{gathered}
A_{1}=\left[\begin{array}{cc}
0.8227 & -0.00168 \\
6.1233 & 0.9367
\end{array}\right], \\
A_{2}=\left[\begin{array}{cc}
0.9654 & -0.00182 \\
-0.6759 & 0.9433
\end{array}\right], \\
A_{3}=\left[\begin{array}{cc}
0.8895 & -0.00294 \\
2.9447 & 0.9968
\end{array}\right], \\
A_{4}=\left[\begin{array}{cc}
0.8930 & -0.00062 \\
2.7738 & 0.8864
\end{array}\right], \\
B_{1}=\left[\begin{array}{c}
-0.000092 \\
0.1014
\end{array}\right], \quad B_{2}=\left[\begin{array}{c}
-0.000097 \\
0.1016
\end{array}\right], \\
B_{3}=\left[\begin{array}{c}
-0.000157 \\
0.1045
\end{array}\right], \quad B_{4}=\left[\begin{array}{c}
-0.000034 \\
0.0986
\end{array}\right],
\end{gathered}
$$




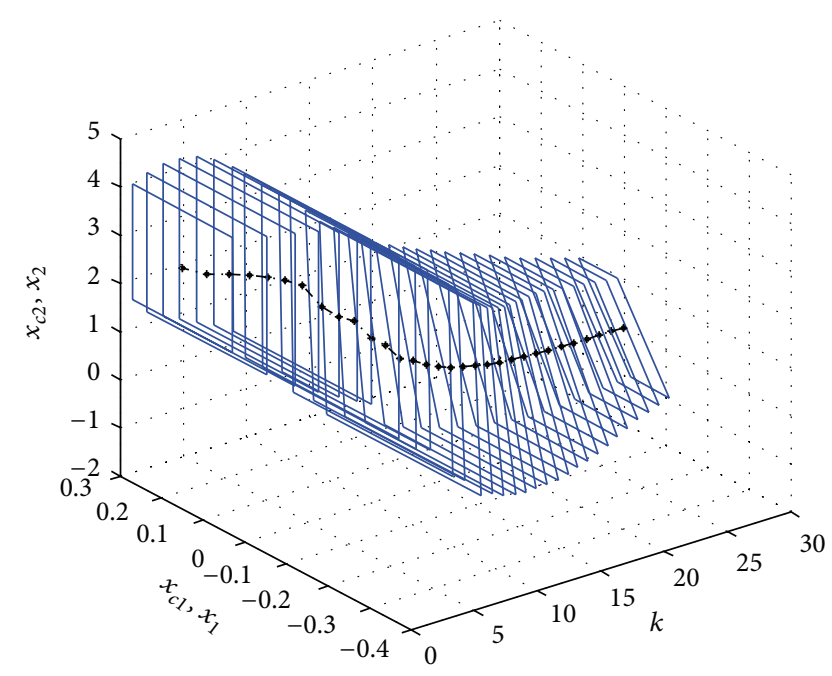

FIGURE 2: The responses of estimated states and time-varying estimation error sets, Algorithm 1 in [16].

$$
\begin{gathered}
D_{l}=\left[\begin{array}{l}
0.0022 \\
0.0564
\end{array}\right], \quad C_{l}=\left[\begin{array}{ll}
0 & 1
\end{array}\right], \quad E_{l}=0.2, \\
l=1, \ldots, 4, \\
\bar{u}=10, \quad \Psi=1, \quad \bar{\psi}=10, \\
\lambda_{1}=\frac{1}{2} \frac{\varphi_{1}(y)-\varphi_{1}(-\bar{\psi})}{\varphi_{1}(\bar{\psi})-\varphi_{1}(-\bar{\psi})}, \quad \lambda_{2}=\frac{1}{2} \frac{\varphi_{1}(\bar{\psi})-\varphi_{1}(y)}{\varphi_{1}(\bar{\psi})-\varphi_{1}(-\bar{\psi})}, \\
\varphi_{1}(y)=7.2 \times 10^{10} e^{-8750 /(y+350)}, \\
\frac{1}{2} \frac{\varphi_{2}(y)-\varphi_{2}(-\bar{\psi})}{\varphi_{2}(\bar{\psi})-\varphi_{2}(-\bar{\psi})}, \quad \lambda_{4}=\frac{1}{2} \frac{\varphi_{2}(\bar{\psi})-\varphi_{2}(y)}{\varphi_{2}(\bar{\psi})-\varphi_{2}(-\bar{\psi})}, \\
\varphi_{2}(y)=\frac{3.6 \times 10^{10}\left(e^{-8750 /(y+350)}-e^{-8750 / 350}\right)}{y} .
\end{gathered}
$$

For the above quasi-LPV system, in Algorithm 8, choose $P_{w}=4, H_{w}=0.5, \mathbb{Q}=25$, and $\mathscr{R}=1$, take $x_{c}(0)=[0.15,3]^{\mathrm{T}}$, and the initial state belongs to a zonotope $x_{c}(0) \oplus H(0) \mathbf{B}^{m(0)}$, where $H(0)=\left[\begin{array}{cc}0.12 & 0 \\ 0 & 1.2\end{array}\right], m(0)=2$. In order to compare with Algorithm 1 in [16], $N_{z}=2$ $\left(V_{X(k)}=V_{P(k)}=4\right)$ is selected in Algorithm 8, and choose the parameters for Algorithm 1 in $[16]$ as $\bar{e}=[0.12,1.2]^{\mathrm{T}}$, $\{\bar{\tau}, \delta, \mu\}=\{10,0.003,0.99\}$. For comparing with Algorithm 1 in [19], $N_{z}=4\left(V_{X(k)}=V_{P(k)}=8\right)$ is selected in Algorithm 8, and choose the parameters for Algorithm 1 in $[19]$ as $\bar{e}=[0.12,1.2]^{\mathrm{T}}, T=\left[\begin{array}{cccc}0.1697 & 0.1697 & 0 & -1.697 \\ 0 & 0.1697 & 1.697 & 1.697\end{array}\right]^{\mathrm{T}}, M_{e}(0)=$ $\operatorname{diag}\left\{0.5 /(0.06 \times 2)^{2}, 0.5 /(0.6 \times 2)^{2}\right\}, \widehat{p}=8$.

For all the compared algorithms above, the disturbance sequence $w$ is randomly generated from the interval $[-0.5,0.5]$, which can be represented by a zonotope $\mathbb{D}=$ $0 \oplus H_{w} \mathbf{B}^{1}$. We have fixed $\alpha=0.001$, which only has

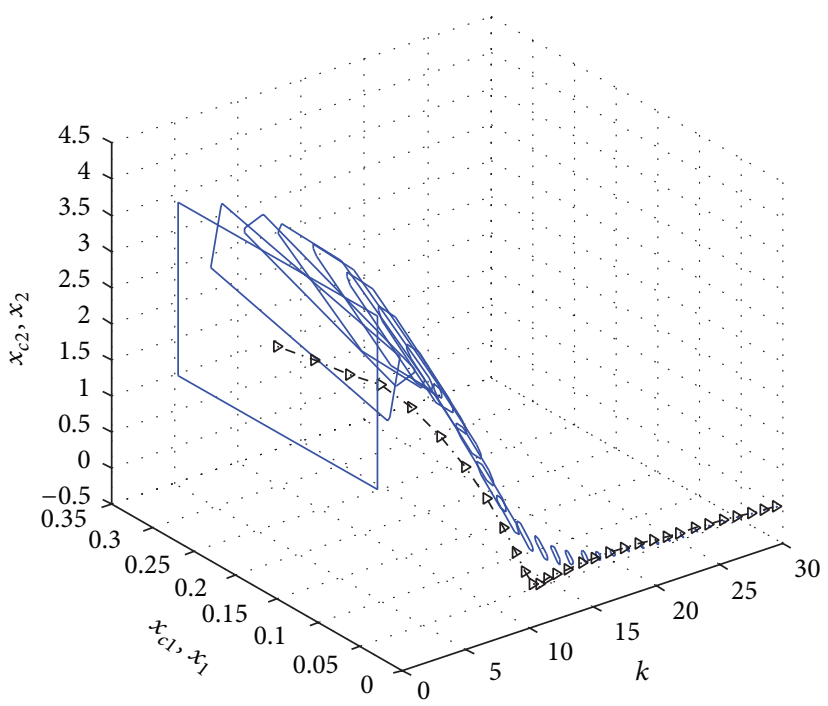

FIGURE 3: The responses of estimated states and time-varying estimation error sets, Algorithm 1 in [19].

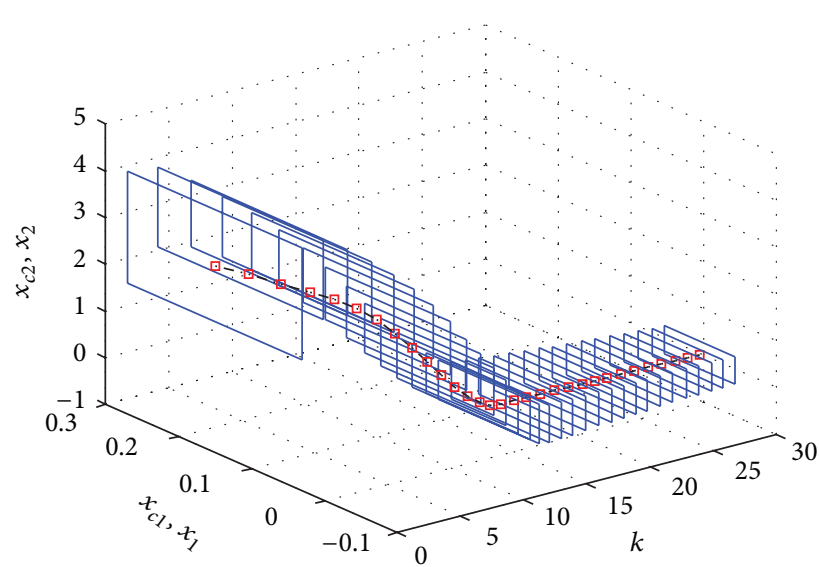

FIGURE 4: The responses of estimated states and time-varying estimation error sets, Algorithm 8, $N_{z}=2$.

negligible influence on the control performance. By applying Algorithm $8\left(N_{z}=2\right)$, Algorithm $8\left(N_{z}=4\right)$, Algorithm 1 in [16], and Algorithm 1 in [19], the simulation results are shown in Figures 2, 3, 4, 5, 6, 7, 8, 9, 10, and 11. Figures 24 show the responses of estimated states and time-varying estimation error sets related to the compared algorithms. Figure 6 compares the trajectories of augmented closed-loop system corresponding to the different algorithms. The control input signals shown in Figure 7 illustrate that the input constraints are satisfied. Figure 8 shows the performance cost of the compared algorithms. The same disturbance sequence that is shown in Figure 9 has been applied for all the simulations. Figures 10 and 11 show the projections of a super dimensional ellipsoid $\varepsilon_{Q_{c}^{-1}}$, which is related to performance cost $\gamma_{c}$, on the subspace of estimated state and estimation error set, respectively. 


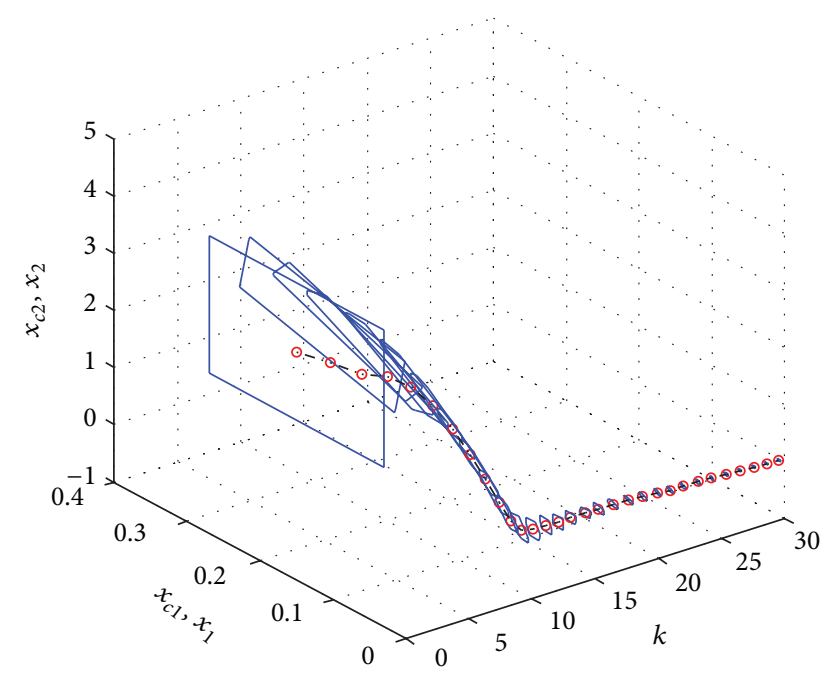

Figure 5: The responses of estimated states and time-varying estimation error sets, Algorithm 8, $N_{z}=4$.

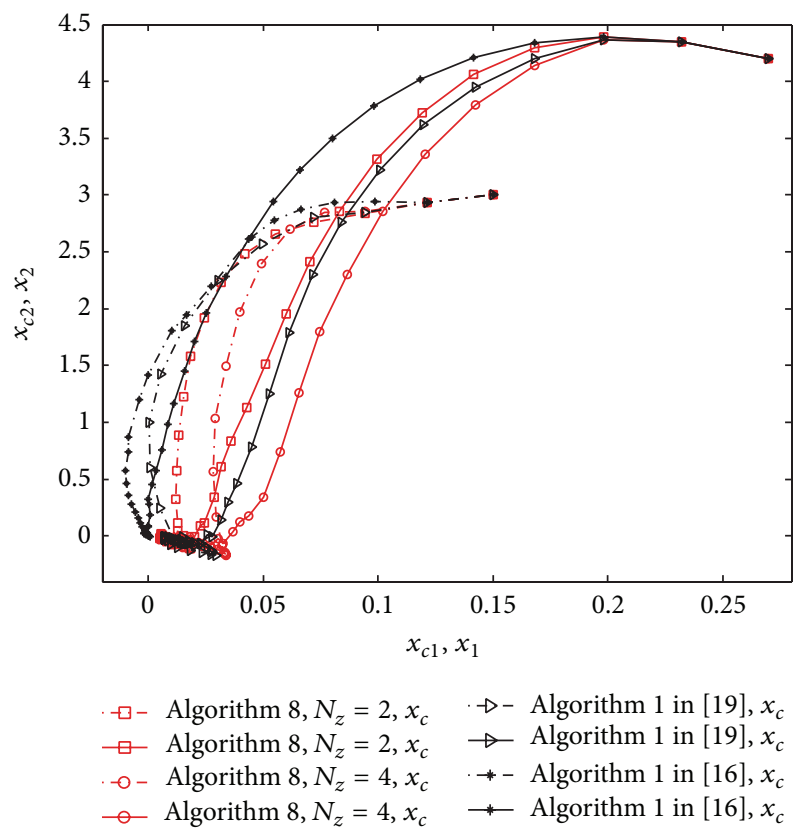

FIGURE 6: The comparisons of trajectories of augmented closed-loop systems.

Let $J=\sum_{k=0}^{30}\left\{\|y(k)\|_{\mathscr{Q}}^{2}+\|u(k)\|_{\mathscr{R}}^{2}\right\}$. For Algorithm 8 $\left(N_{z}=2\right)$ (Algorithm $8\left(N_{z}=4\right)$, Algorithm 1 in [16], and Algorithm 1 in [19]), $J=3808.8(J=3374.5, J=$ $4253.6, J=3658.8)$ is obtained, and the time spent on the simulations is $36.4 \mathrm{~s}(42.6 \mathrm{~s}, 50.8 \mathrm{~s}, 49.2 \mathrm{~s})$, respectively. Compare Algorithm $8\left(N_{z}=2\right)$ with Algorithm 1 in [16]; the control performance is improved with lower computational burden; even the main optimization problem of Algorithm 1 in [16] is not solved at some sampling times. Compare Algorithm $8\left(N_{z}=2\right)$ with Algorithm $8\left(N_{z}=4\right)$; by properly

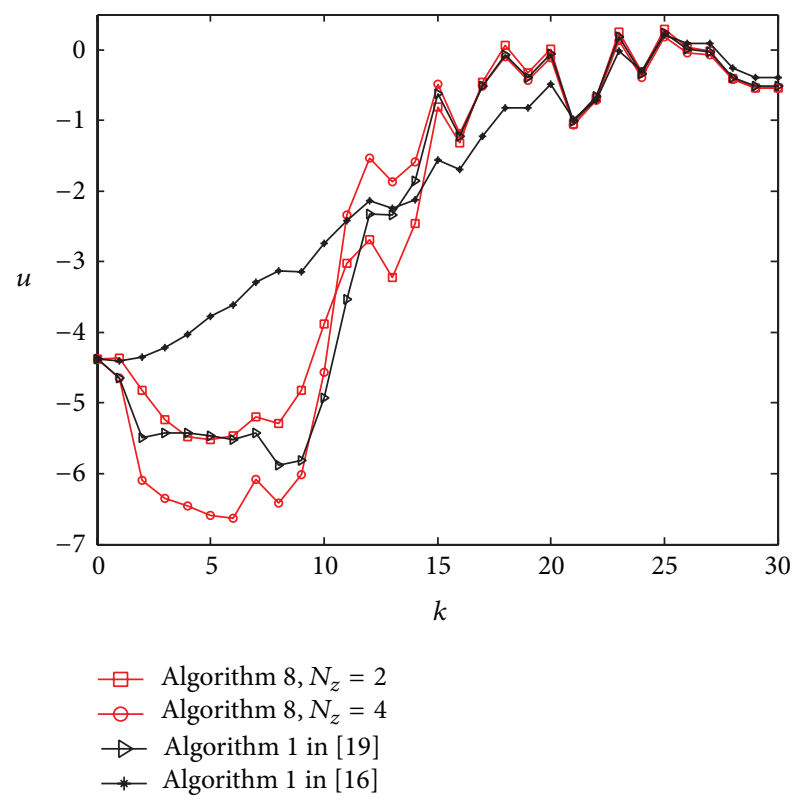

Figure 7: The comparisons of control input signals.

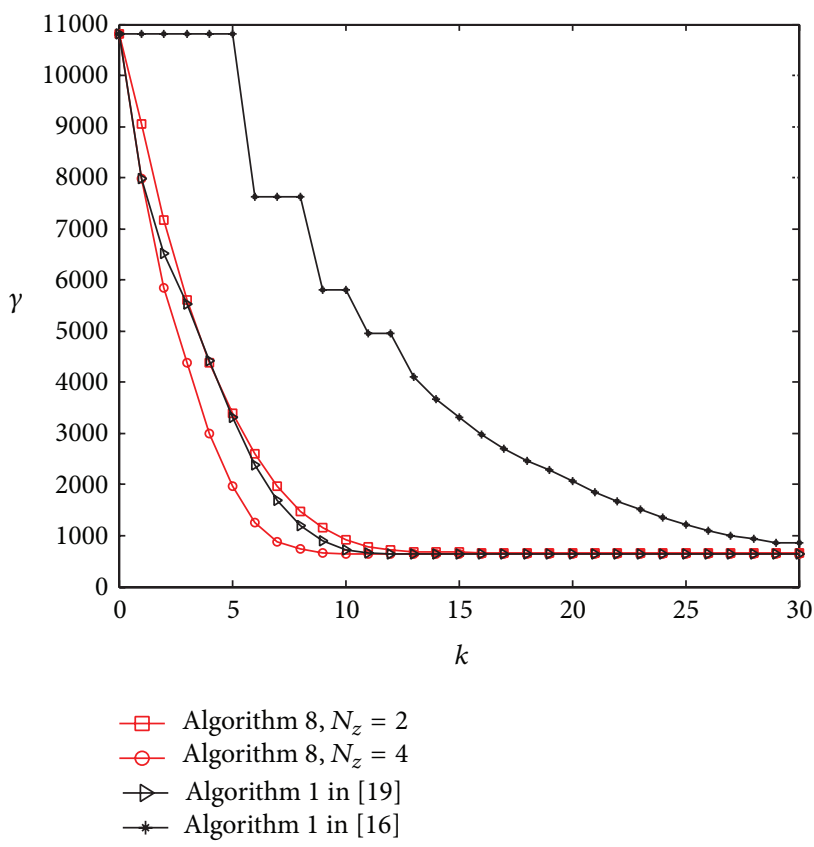

FIGURE 8: The performance costs of the compared algorithms.

limiting the order of zonotopes, the control performance can be improved, with a slight increasement of computational burden. Furthermore, the augmented states of Algorithm 8 $\left(N_{z}=4\right)$ and Algorithm 1 in [19] converge to nearly the same neighborhoods of $\tilde{x}=0$, while the control performance of the former is better than the latter. The Matlab 7.14 (AMD Phenom II Processor 2.70 GHz, 4 G Memory) is utilized for the simulations. 


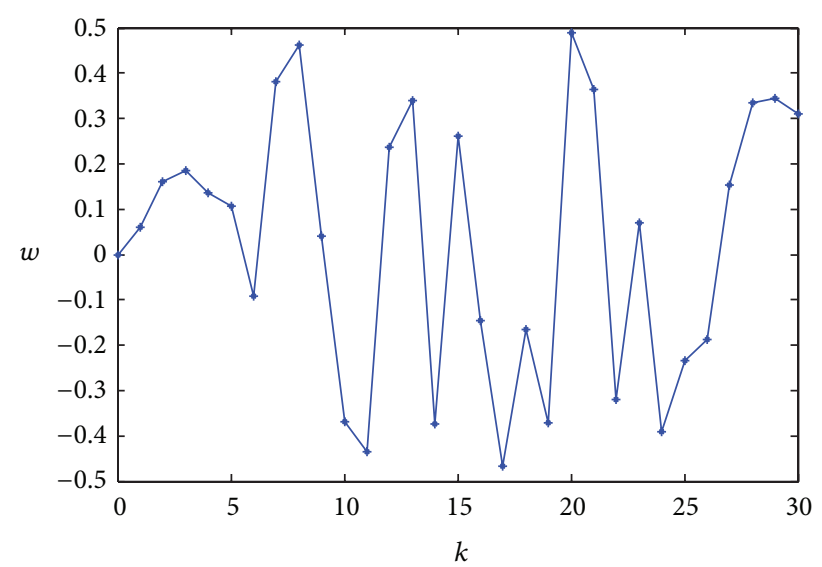

FIGURE 9: The disturbance utilized in the simulations.

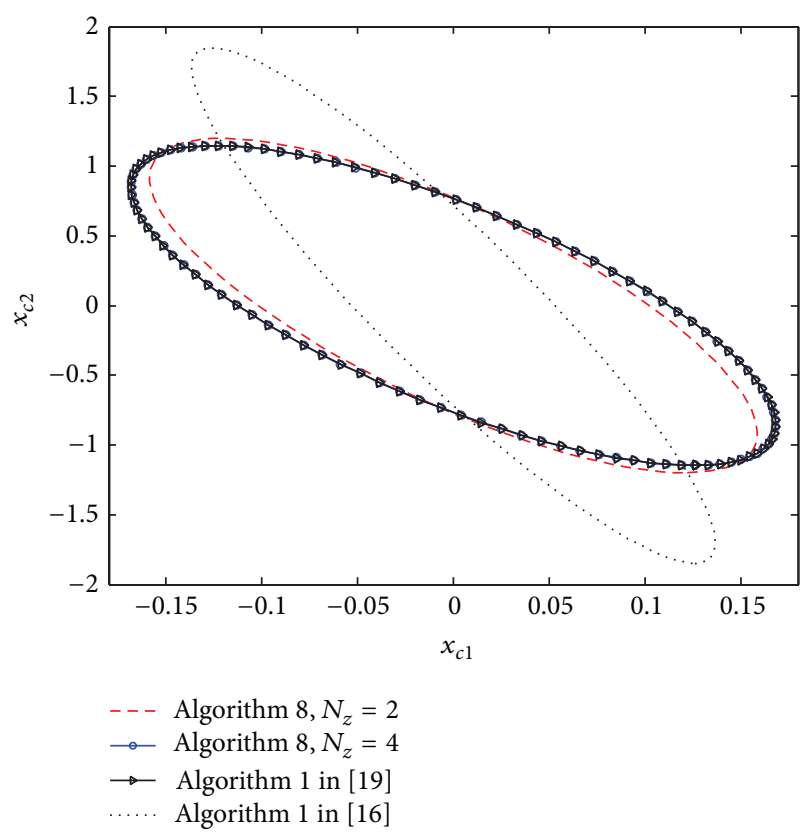

FIGURE 10: The projection of $\varepsilon_{Q_{c}^{-1}}$ on the subspace of estimated state.

\section{Conclusions}

For quasi-LPV systems with bounded disturbance, a setmembership estimation method via zonotopic computation is applied to refresh the estimation error set. By properly refreshing the estimation error set, the control performance can be improved. Furthermore, the feasibility of the main optimization problem at the next sampling time can be determined by checking a feasible problem at the current time. The augmented state can converge to a neighborhood of $\widetilde{x}=0$ and stay in the neighborhood of $\widetilde{x}=0$ thereafter.

\section{Conflict of Interests}

The authors declare that there is no conflict of interests regarding the publication of this paper.

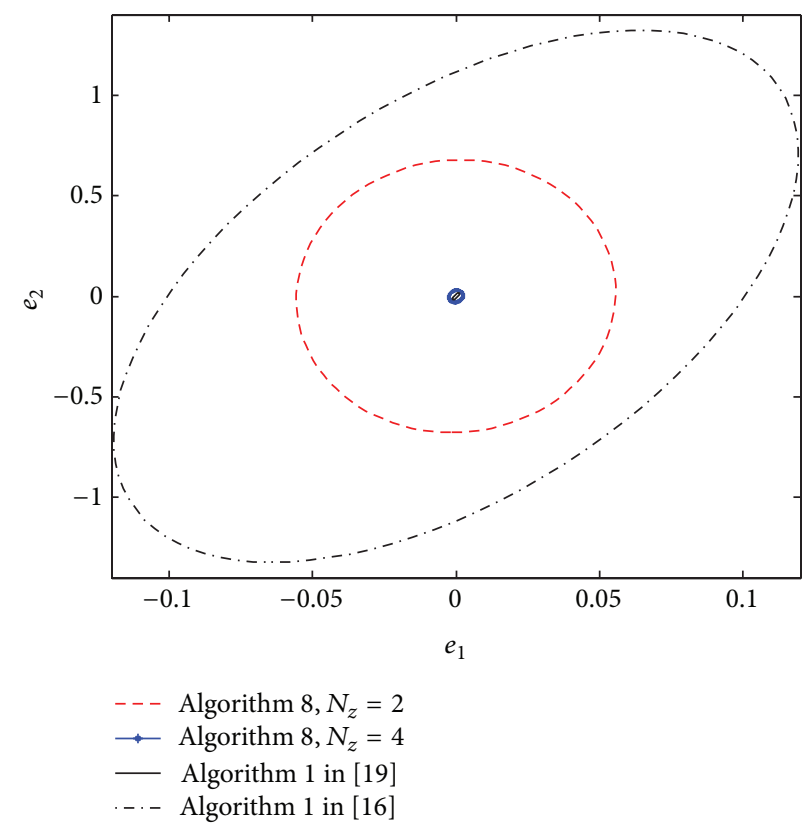

FIGURE 11: The projection of $\varepsilon_{\mathrm{Q}_{c}^{-1}}$ on the subspace of estimation error.

\section{Acknowledgments}

This work is supported by the National Nature Science Foundation of China (NSFC, 61403297 and 61370037) and the Fundamental Research Funds for the Central Universities (K5051304038, JB140417).

\section{References}

[1] D. Q. Mayne, J. B. Rawlings, C. V. Rao, and P. O. Scokaert, "Constrained model predictive control: stability and optimality," Automatica, vol. 36, no. 6, pp. 789-814, 2000.

[2] M. L. Darby and M. Nikolaou, "MPC: current practice and challenges," Control Engineering Practice, vol. 20, no. 4, pp. 328342, 2012.

[3] S. J. Qin and T. A. Badgwell, "A survey of industrial model predictive control technology," Control Engineering Practice, vol. 11, no. 7, pp. 733-764, 2003.

[4] A. S. Kumar and Z. Ahmad, "Model predictive control (MPC) and its current issues in chemical engineering," Chemical Engineering Communications, vol. 199, no. 4, pp. 472-511, 2012.

[5] M. V. Kothare, V. Balakrishnan, and M. Morari, "Robust constrained model predictive control using linear matrix inequalities," Automatica, vol. 32, no. 10, pp. 1361-1379, 1996.

[6] Y. Lu and Y. Arkun, "Quasi-min-max MPC algorithms for LPV systems," Automatica, vol. 36, no. 4, pp. 527-540, 2000.

[7] D. Li and Y. Xi, "The feedback robust MPC for LPV systems with bounded rates of parameter changes," IEEE Transactions on Automatic Control, vol. 55, no. 2, pp. 503-507, 2010.

[8] M. Jungers, R. C. Oliveira, and P. Peres, "MPC and LPV systems with bounded parameter variations," International Journal of Control, vol. 84, no. 1, pp. 24-36, 2011.

[9] S. Yu, C. Böhm, H. Chen, and F. Allgöwer, "Model predictive control of constrained LPV systems," International Journal of Control, vol. 85, no. 6, pp. 671-683, 2012. 
[10] D. Q. Mayne, S. V. Raković, R. Findeisen, and F. Allgöwer, "Robust output feedback model predictive control of constrained linear systems," Automatica, vol. 42, no. 7, pp. 12171222, 2006.

[11] D. Q. Mayne, S. V. Raković, R. Findeisen, and F. Allgöwer, "Robust output feedback model predictive control of constrained linear systems: time varying case," Automatica, vol. 45, no. 9, pp. 2082-2087, 2009.

[12] Z. Wan and M. V. Kothare, "Robust output feedback model predictive control using off-line linear matrix inequalities," Journal of Process Control, vol. 12, no. 7, pp. 763-774, 2002.

[13] D. Li, Y. Xi, and F. Gao, "Synthesis of dynamic output feedback RMPC with saturated inputs," Automatica, vol. 49, no. 4, pp. 949-954, 2013.

[14] B. Ding and X. Ping, "Output feedback predictive control with one free control move for nonlinear systems represented by a takagi-sugeno model," IEEE Transactions on Fuzzy Systems, vol. 22, no. 2, pp. 249-263, 2014.

[15] B. Ding, Y. Xi, M. T. Cychowski, and T. O’Mahony, "A synthesis approach for output feedback robust constrained model predictive control," Automatica, vol. 44, no. 1, pp. 258-264, 2008.

[16] B. Ding, "Constrained robust model predictive control via parameter-dependent dynamic output feedback," Automatica, vol. 46, no. 9, pp. 1517-1523, 2010.

[17] D. Famularo and G. Franzè, "Output feedback model predictive control of uncertain norm-bounded linear systems," International Journal of Robust and Nonlinear Control, vol. 21, no. 8, pp. 838-862, 2011.

[18] B. Ding, "Dynamic output feedback predictive control for nonlinear systems represented by a Takagi-Sugeno model," IEEE Transactions on Fuzzy Systems, vol. 19, no. 5, pp. 831-843, 2011.

[19] B. Ding, X. Ping, and H. Pan, "On dynamic output feedback robust MPC for constrained quasi-LPV systems," International Journal of Control, vol. 86, no. 12, pp. 2215-2227, 2013.

[20] X. Ping and B. Ding, "Off-line approach to dynamic output feedback robust model predictive control," Systems and Control Letters, vol. 62, no. 11, pp. 1038-1048, 2013.

[21] E. Walter and H. Piet-Lahanier, "Exact recursive polyhedral description of the feasible parameter set for bounded-error models," IEEE Transactions on Automatic Control, vol. 34, no. 8, pp. 911-915, 1989.

[22] C. Combastel, "A state bounding observer based on zonotopes," in Proceedings of European Control Conference, Cambridge, UK, 2003.

[23] T. Alamo, J. M. Bravo, and E. F. Camacho, "Guaranteed state estimation by zonotopes," Automatica, vol. 41, no. 6, pp. 10351043, 2005.

[24] T. Alamo, J. M. Bravo, M. J. Redondo, and E. Camacho, "A set-membership state estimation algorithm based on DC programming," Automatica, vol. 44, no. 1, pp. 216-224, 2008.

[25] V. T. H. Le, T. Alamo, E. F. Camacho, C. Stoica, and D. Dumur, "A new approach for guaranteed state estimation by zonotopes," in Proceedings of the 18th IFAC World Congress, pp. 9242-9247, Milano, Italy, September 2011.

[26] C. B. Barber, D. P. Dobkin, and H. Huhdanpaa, "The quickhull algorithm for convex hulls," ACM Transactions on Mathematical Software, vol. 22, no. 4, pp. 469-483, 1996.

[27] S. Schön and H. Kutterer, "Using zonotopes for overestimationfree interval least-squares-some geodetic applications," Reliable Computing, vol. 11, no. 2, pp. 137-155, 2005.
[28] K. Fukuda, "From the zonotope construction to the Minkowski addition of convex polytopes," Journal of Symbolic Computation, vol. 38, no. 4, pp. 1261-1272, 2004.

[29] A. Alessandri, M. Baglietto, and G. Battistelli, "On estimation error bounds for receding-horizon filters using quadratic boundedness," IEEE Transactions on Automatic Control, vol. 49, no. 8, pp. 1350-1355, 2004.

[30] A. Alessandri, M. Baglietto, and G. Battistelli, "Design of state estimators for uncertain linear systems using quadratic boundedness," Automatica, vol. 42, no. 3, pp. 497-502, 2006.

[31] P. Gahinet, A. Nemirovski, A. J. Laub et al., LMI Control Toolbox for Use with Matlab, Users Guide, The Math Works Inc., Natick, Mass, USA, 1995.

[32] B. Ding, "New formulation of dynamic output feedback robust model predictive control with guaranteed quadratic boundedness," Asian Journal of Control, vol. 15, no. 1, pp. 302-309, 2013. 


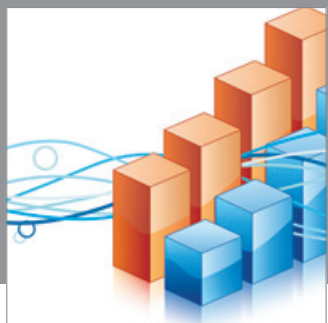

Advances in

Operations Research

mansans

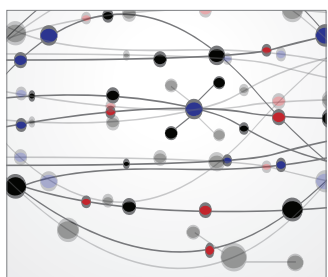

The Scientific World Journal
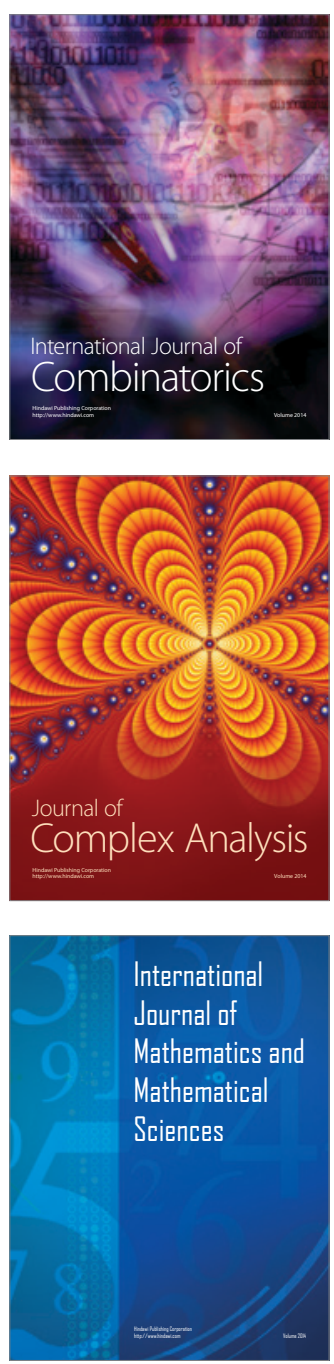
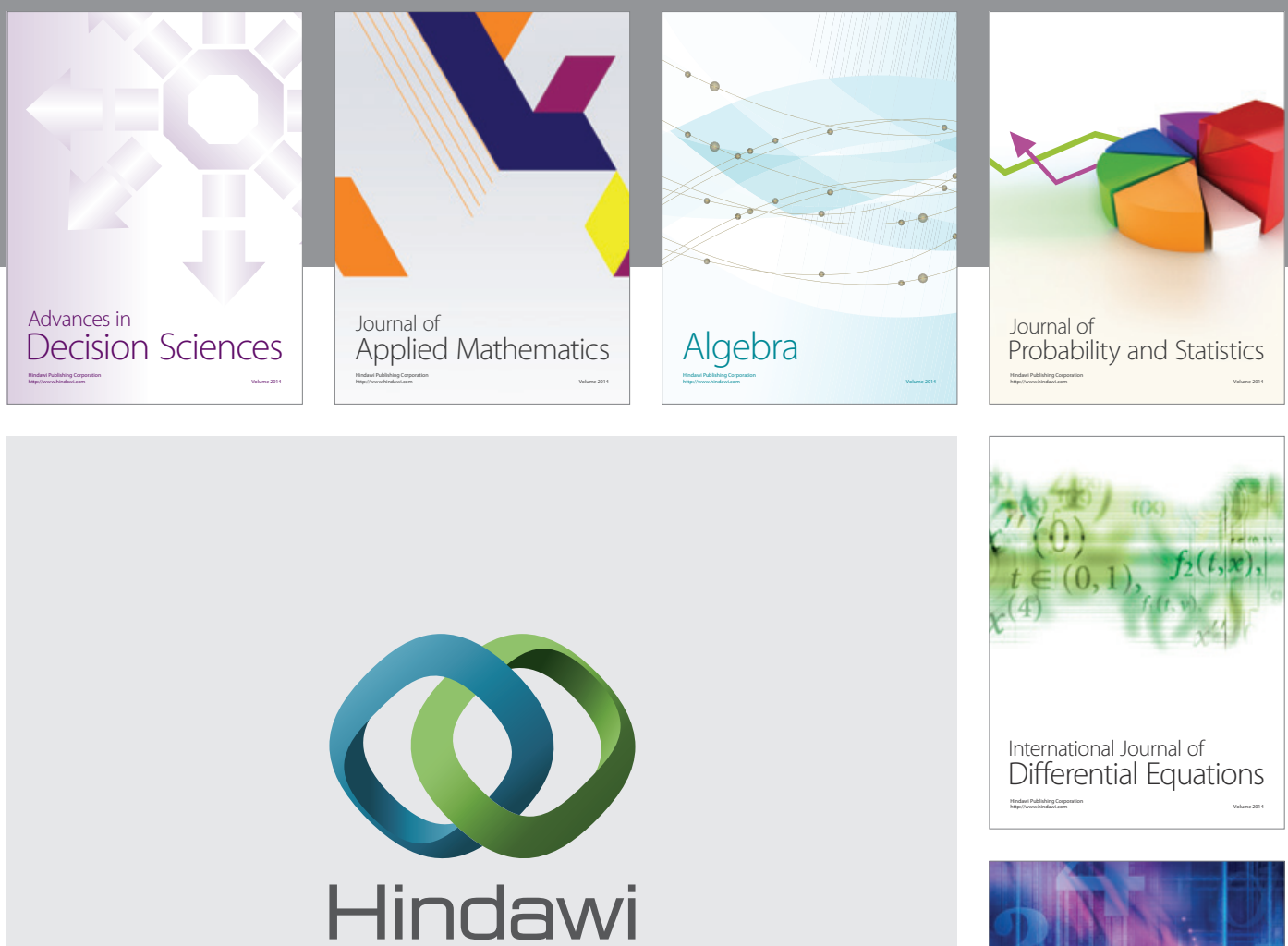

Submit your manuscripts at http://www.hindawi.com
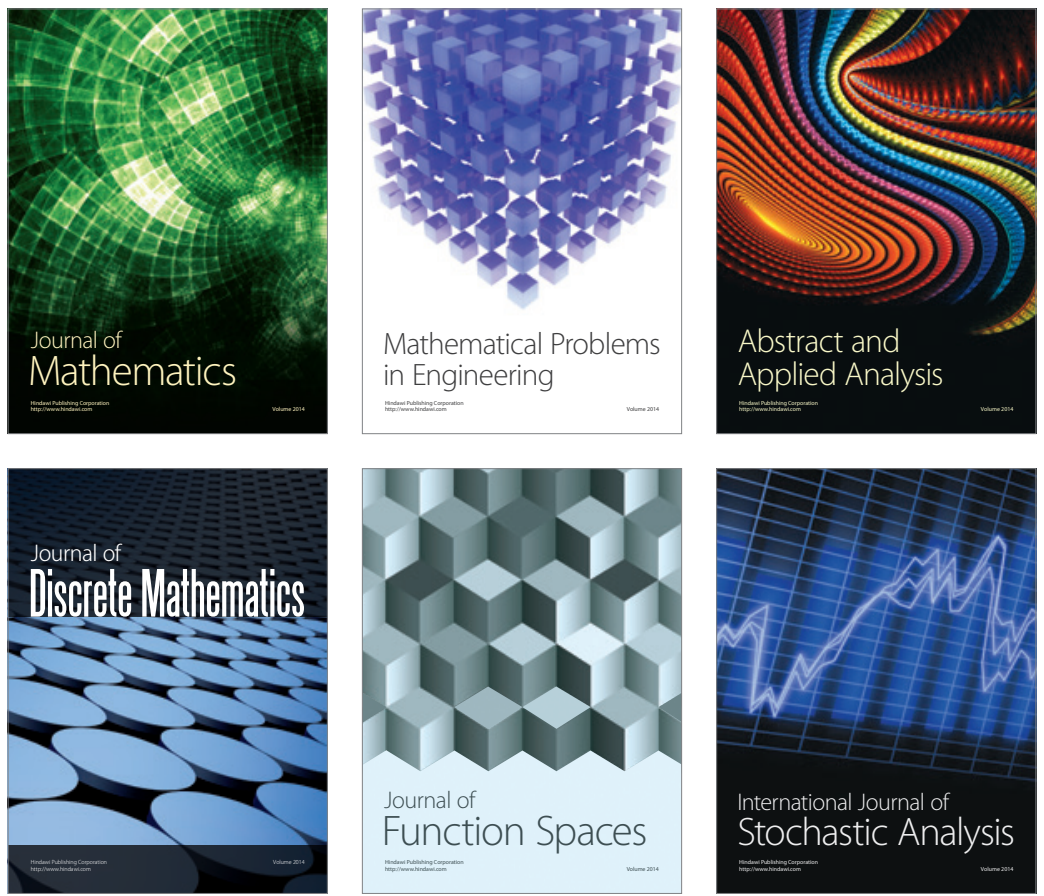

Journal of

Function Spaces

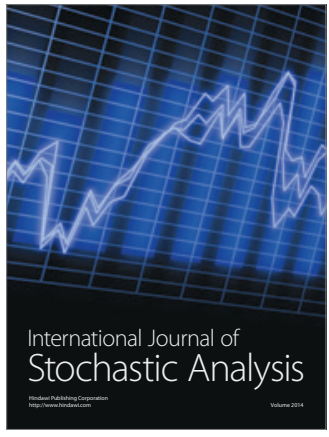

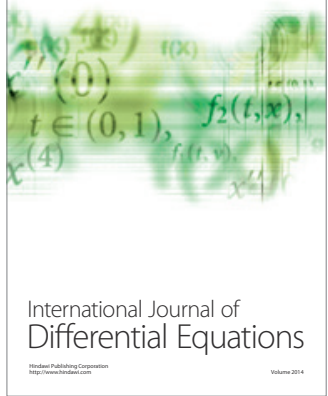
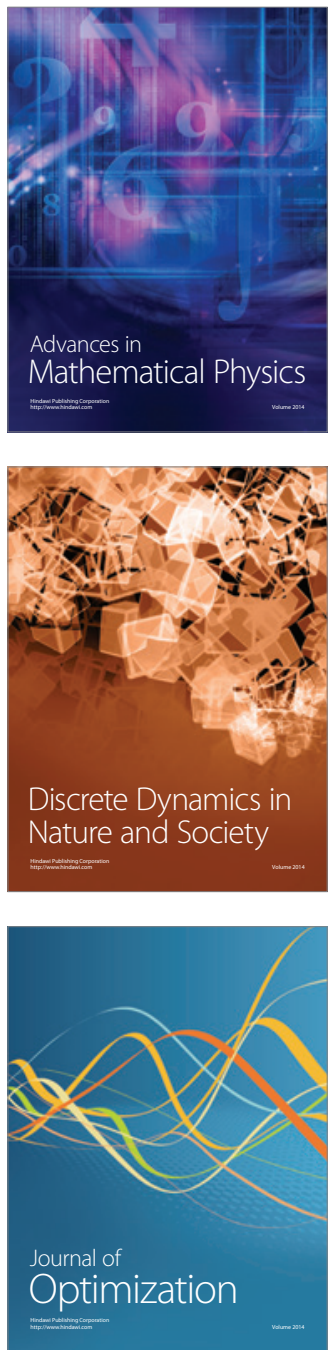